\title{
Nachrufe
}

Gefässchirurgie 2021 $26: 149-150$ https://doi.org/10.1007/s00772-021-00747-3 Angenommen: 8. Januar 2021

Online publiziert: 29. Januar 2021

๑) Springer Medizin Verlag GmbH, ein Teil von Springer Nature 2021
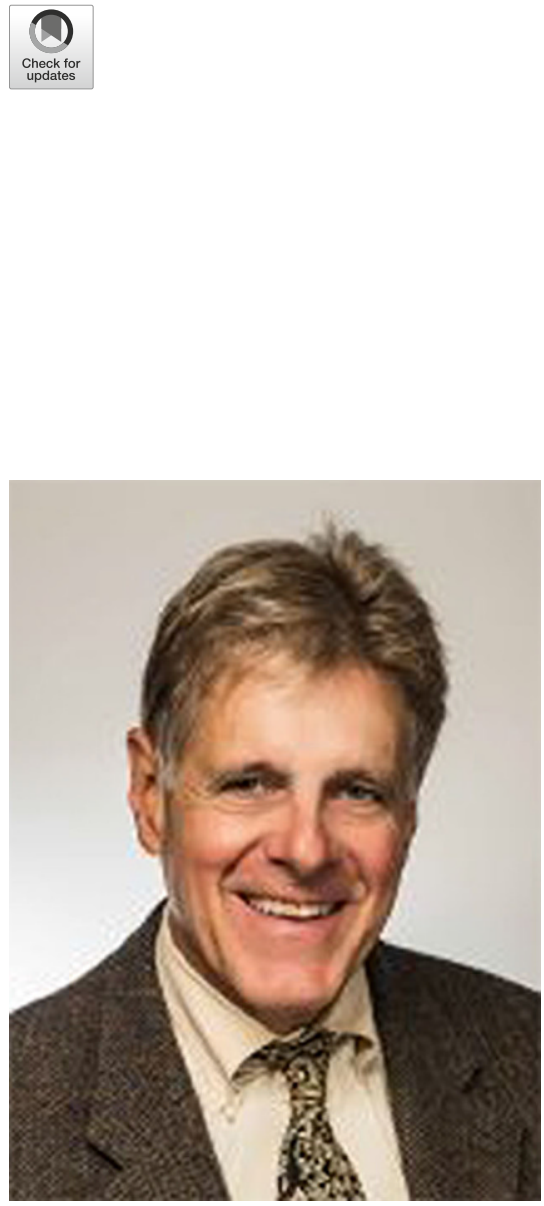

Dr. William R. Hiatt, einer der Väter der vaskulären Medizin in Amerika, ist am 8. Dezember 2020 verstorben. Dr. Hiatt widmete sein Leben der Verbesserung der Versorgung von Gefäßpatienten. Mit seinem Tod verlieren wir eine der Säulen der klinischen Gefäßmedizin und der kardiovaskulären Forschung sowie einen engagierten Mentor für viele von uns. Er hat sein Wissen uns allen gewidmet, unabhängig davon, ob Kardiologe, Angiologe, Herz- oder Gefäßchirurg oder ob jemand ausschließlich Forscher war, zunächst aber den Patienten.

William R. Hiatt wurde 1950 in Colorado geboren. Sein Vater war Zahnarzt, Wissenschaftler und Gründer der University of Colorado Dental School und ein begeisterter Sportler und Bergsteiger. Er war ein Vorbild für Will, der die

\author{
E. S. Debus ${ }^{1} \cdot$ R. Bauersachs ${ }^{2} \cdot$ M. P. Bonaca ${ }^{3} \cdot$ R. T. Grundmann ${ }^{4}$ \\ ' Universitäres Herz- und Gefäßzentrum UKE Hamburg GmbH, Universitätsklinikum Hamburg-Eppendorf, \\ Hamburg, Deutschland \\ ${ }^{2}$ Klinik für Gefäßmedizin, Klinikum Darmstadt, Darmstadt, Deutschland \\ ${ }^{3}$ University of Colorado School of Medicine, Aurora, USA \\ ${ }^{4}$ Burghausen, Deutschland
}

\section{William R. Hiatt, MD}

\section{In memoriam - \\ 1. Juni 1950 - 8. Dezember 2020}

Grundwerte seines Vaters übernahm, anderen durch Wissenschaft und medizinische Versorgung zu helfen, was letztlich seine Karriere bestimmte. Er teilte auch den Abenteuergeist seines Vaters und wurde ein begeisterter und erfolgreicher Sportler und Naturliebhaber. Er bestieg alle 54 „Fourteeners“ (Berge über 14.000 Fuß) in Colorado mindestens einmal. Als Familienmensch fand Will seine größte Freude darin, Zeit mit seiner $\mathrm{Fa}$ milie $\mathrm{zu}$ verbringen.

Dr. Hiatt absolvierte sein akademisches Examen an der University of ColoradoSchool of Medicine. Er bestand seine Facharztausbildung in innerer Medizin am Universitätskrankenhaus in Boston und schloss ein Fellowship in Gefäßmedizin an der Universität von Colorado ab. An der University of Colorado wurde er Professor für Medizin, wo er den Novartis Foundation Endowed Chair in Cardiovascular Research innehatte.

Dr. Hiatt widmete seine Karriere insbesondere der Versorgung von Patienten mit peripherer arterieller Verschlusskrankheit. Er war ehemaliger Präsident der Society of Vascular Medicine. Darüber hinaus war er Vorsitzender des American Heart Association's Council on Peripheral Vascular Disease. Er war außerdem Mitherausgeber der TASCRichtlinien (Inter-Society Consensus for the Management of Peripheral Arterial Disease) und arbeitete unermüdlich daran, multidisziplinäre Empfehlungen für die Versorgung von Patienten mit Gefäßerkrankungen zu erstellen. Während seiner Karriere erhielt er zahlreiche
Auszeichnungen und Anerkennungen für seine Arbeit. Dazu gehörten die Ernennung zum Master der Society for Vascular Medicine und die Distinguished Scientist und Clinical Research Scientist Awards der American Heart Association.

Er erkannte, dass er den größten Einfluss auf die Ergebnisse für die Patienten hatte, wenn er nicht nur Menschen in der Klinik betreute, sondern auch die kardiovaskuläre Forschung leitete und zur regulatorischen Arbeit bezüglich kardiovaskulärer Medikamente beitrug: Er war Mitglied in mehreren FDA-Beratungskomitees und Vorsitzender des US Food and Drug Administration Cardiovascular and Renal Division Advisory Committee. Für diesen Dienst wurde er mit dem FDA Advisory Committee Service Award, Food and Drug Administration (2008) ausgezeichnet.

Will Hiatt hatte globalen Einfluss, aber er blieb immer an der University of Colorado beheimatet, wo er in zahlreichen Positionen eine herausragende Führungsrolle einnahm. Er veröffentlichte über 280 Manuskripte, darunter wegweisende Publikationen zur Bewertung optimaler Therapien bei peripherer Arterienerkrankung, und er verfasste über 60 Buchkapitel. Er war Lehrer und Mentor zahlreicher ärztlicher Wissenschaftler und versorgte unzählige Patienten mit komplexen kardiovaskulären Erkrankungen. Will Hiatt widmete sich intensiv physiologischen Studien und wurde ein Meister der Messung funktioneller Ergebnisse. Seine Mission zur Verbesserung der Ergebnisse bei pe- 
ripherer Arterienerkrankung erstreckte sich über Früh- und Spätphasenstudien, Beobachtungsstudien, Bevölkerungsstudien und Registerstudien. Seine Arbeit führte zur kollaborativen Leitung der EUCLID-Studie (Hiatt et al. NEJM 2017) mit dem Duke Clinical Research Institute, der bislang größten dedizierten Studie zur medizinischen Therapie der peripheren arteriellen Verschlusskrankheit. Eine seiner größten und letzten Leistungen aber war die Leitung und Durchführung der VOYAGER PAD Studie (Bonaca et al. NEJM 2020) als neuartige Outcome-Studie bei peripherer arterieller Verschlusskrankheit (pAVK). Diese Studie hat einen entscheidenden Einfluss auf die Reduzierung schwerer kardialer und Extremitätenereignisse bei Patienten mit peripherer Arterienerkrankung, die eine Revaskularisation der unteren Extremitäten benötigen. Er konnte diese wegweisende Studie gerade noch vor seinem Tod beenden. Das Vermächtnis seines Engagements für die Versorgung von Patienten mit pAVK hat entscheidend dazu beigetragen, die Forschung in diesem Bereich zu verändern und verspricht, die Ergebnisse in dieser Hochrisikogruppe zu verbessern. Er ist damit ein wesentlicher Wegbereiter für die evidenzbasierte Therapie dieser
Erkrankung und hat hier entscheidende Impulse gesetzt.

Will Hiatt war nicht nur ein bemerkenswerter Arzt und Wissenschaftler, er war auch ein bemerkenswerter Mensch. Er war ungewöhnlich geerdet, bescheiden, dankbar und großzügig. Will kümmerte sich nicht um Beruf, Herkunft und sozialen Status seiner Gesprächspartner. Jeder, unabhängig von seinem Beruf, war bei ihm herzlich willkommen, und er war im wahrsten Sinne des Wortes ein großer Mentor für alle, die wir das Privileg hatten, mit ihm arbeiten und seine Gedanken mit ihm teilen zu können. Für viele von uns war er ein verlässlicher Freund. Will hatte einen Sinn für eine höhere Mission - über unsere täglichen Aufgaben hinaus. Und das einte die, die ähnlich dachten. Sein hoher Sinn für ethische Haltung ist im Code of the West zusammengefasst (im Original von James P. Owen in Cowboy Ethics, 2005):

Lebe jeden Tag mit Mut

Sei stolz auf deine Arbeit

Beende immer, was du anfängst

Tu, was getan werden muss

Sei hart, aber fair

Wenn Du ein Versprechen gibst, halte es, Stehe für das ein, wovon Du überzeugt bist,

Rede weniger und sage mehr,
Denke daran, dass manche Dinge nicht zum Verkauf stehen,

Sei Dir bewusst, wo man die Grenze ziehen muss

Sebastian Debus,

Rupert Bauersachs,

Marc Bonaca und

Reinhart Grundmann

\section{Korrespondenzadresse}

Prof. Dr. E. S. Debus

Universitäres Herz- und Gefäßzentrum UKE Hamburg GmbH, Universitätsklinikum Hamburg-Eppendorf

Martinistr. 52, 20246 Hamburg, Deutschland s.debus@uke.de
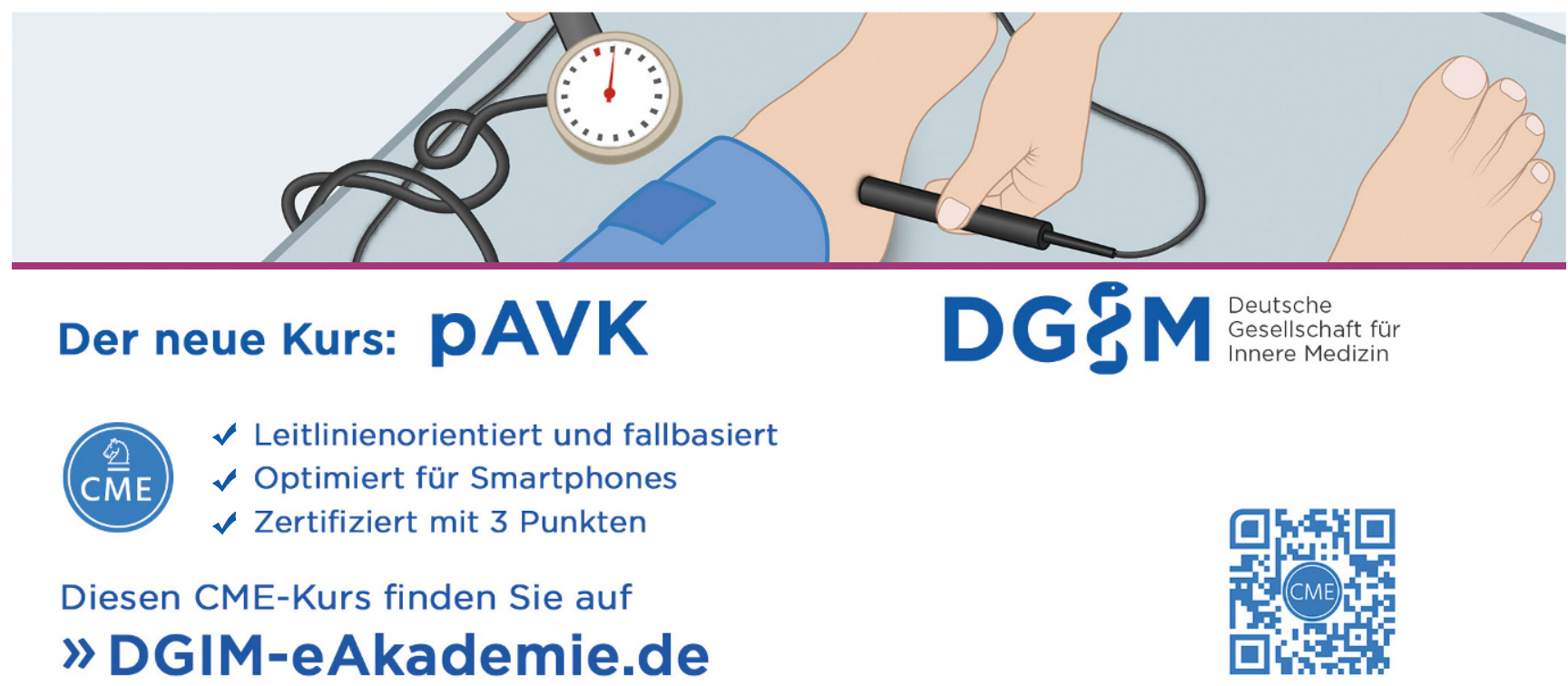

Das Fortbildungs-Portal der DGIM: Kostenfrei für alle Mitglieder und e.Med-Abonnenten 